\title{
mTOR inhibition and BMP signaling act synergistically to reduce muscle fibrosis and improve myofiber regeneration
}

\author{
Shailesh Agarwal,' David Cholok, ${ }^{1}$ Shawn Loder, ${ }^{1}$ John Li, ${ }^{1}$ Christopher Breuler, ${ }^{1}$ Michael T. Chung, ${ }^{1}$ \\ Hsiao Hsin Sung, ' Kavitha Ranganathan, ${ }^{1}$ Joe Habbouche, ${ }^{1}$ James Drake, ${ }^{1}$ Joshua Peterson, ${ }^{1}$ \\ Caitlin Priest, ${ }^{1}$ Shuli Li, ${ }^{1}$ Yuji Mishina, ${ }^{2}$ and Benjamin Levi ${ }^{1}$ \\ 'Department of Surgery, University of Michigan Health System, Ann Arbor, Michigan, USA. ${ }^{2}$ School of Dentistry, University \\ of Michigan, Ann Arbor, Michigan, USA.
}

\begin{abstract}
Muscle trauma is highly morbid due to intramuscular scarring, or fibrosis, and muscle atrophy. Studies have shown that bone morphogenetic proteins (BMPs) reduce muscle atrophy. However, increased BMP signaling at muscle injury sites causes heterotopic ossification, as seen in patients with fibrodysplasia ossificans progressiva (FOP), or patients with surgically placed BMP implants for bone healing. We use a genetic mouse model of hyperactive BMP signaling to show the development of intramuscular fibrosis surrounding areas of ectopic bone following muscle injury. Rapamycin, which we have previously shown to eliminate ectopic ossification in this model, also eliminates fibrosis without reducing osteogenic differentiation, suggesting clinical value for patients with FOP and with BMP implants. Finally, we use reporter mice to show that BMP signaling is positively associated with myofiber cross-sectional area. These findings underscore an approach in which 2 therapeutics (rapamycin and BMP ligand) can offset each other, leading to an improved outcome.
\end{abstract}

Authorship note: S. Agarwal and D. Cholok contributed equally to this work.

Conflict of interest: The authors declare that no conflict of interest exists.

Submitted: July 28, 2016

Accepted: October 25, 2016

Published: December 8, 2016

Reference information: JCI Insight. 2016;1(20):e89805. doi:10.1172/jici.nsight.89805.

\section{Introduction}

Muscle injury following trauma leads to loss of function as a result of intramuscular fibrosis and myofiber atrophy $(1,2)$. Previous studies have shown that bone morphogenetic protein (BMP) signaling is positively associated with muscle size (3-5). However, these findings have not been realized in the setting of muscle injury, owing to the robust ectopic bone that forms with local BMP delivery (6-10). Clinically, this robust inflammatory response and ectopic bone is found in patients who have adverse reactions to recombinant human BMP2 (rhBMP2) implants (refs. 10-18 and Supplemental Table 1; supplemental material available online with this article; doi:10.1172/jci.insight.89805DS1). Additionally, patients with fibrodysplasia ossificans progressiva (FOP) harbor a mutated version of the type I BMP receptor (T1-BMPR) ACVR1/ ALK2, which causes catastrophic heterotopic bone at sites of muscle injury $(19,20)$. Therefore, a strategy to eliminate the adverse effects associated with BMP signaling at sites of muscle injury, while achieving the reduced myofiber atrophy seen with injury, would make BMP an attractive clinical option for patients with muscle trauma.

Rapamycin has previously been shown to reduce fibrosis in multiple tissue types including muscle, kidney, liver, and lungs (21-28). We have also previously shown that rapamycin eliminates ectopic bone in a genetic model of hyperactive BMP signaling (29). However, studies have also shown that when administered in models of muscle trauma, rapamycin causes unwanted muscle atrophy $(2,30)$. Therefore, a strategy to eliminate the adverse effects associated with rapamycin and loss of mammalian target of rapamycin (mTOR) at sites of muscle injury, while achieving the reduced fibrosis and pathologic mesenchymal cell presence at sites of muscle injury would make rapamycin an attractive clinical option for patients with muscle trauma.

In this study, we show that in models of local BMP delivery (ossicle) or hyperactive T1-BMPR activity $\left(c_{A} \operatorname{Acvr}^{f / f f}\right)$, areas of myofiber injury and fibrosis precede formation of ectopic bone. Rapamycin reduces the fibrosis and accumulation of mesenchymal cells observed at the injury site of mutant mice, suggesting that this drug has utility in reducing muscle injury in FOP patients. Additionally, rapamycin preserves BMP signaling and osteogenic differentiation, suggesting a potential therapeutic option for patients with 
rhBMP2 implants who are at risk for muscle edema and inflammation but require preservation of the osteogenic properties of BMP. Finally, we show that increased BMP signaling improves myofiber size in the injury site of rapamycin-treated mutant mice. Taken together, these findings indicate that rapamycin and BMP may positively influence muscle healing in the setting of injury while offsetting the adverse consequences associated with each individually.

\section{Results}

Hyperactive BMP signaling causes intramuscular fibrosis in addition to ectopic bone. Ectopic bone formation in FOP is concurrent with local muscle destruction $(31,32)$. However, models of intramuscular BMP injection or hyperactive T1-BMPR signaling are typically only used to study the osseous lesion $(6,7,9)$. Therefore, we first characterized the intramuscular damage present in the context of upregulated BMP signaling. Implantation of a BMP scaffold with concurrent cardiotoxin-induced (CTX-induced) muscle injury leads to robust ectopic bone (Supplemental Figure 1A). In addition, we identified substantial myofiber damage indicated by centralized nuclei (Supplemental Figure 1B) and fibrosis (Supplemental Figure 1C) extending from the osseous lesion.

We next used a model of Cre-inducible hyperactive T1-BMPR signaling (caAcvr1 flff: ACVR1 Q207D) (Figure 1A). This model is more consistent with the biology of FOP in which hyperactive ACVR1/ALK2 signaling drives an exaggerated response to trauma leading to ectopic bone (7). Consistent with previous findings, we obtained ectopic osseous lesions after simultaneous injection of adenoviral Cre (Ad.cre) and CTX (Ad.cre/CTX) into the hindlimb (Supplemental Figure 2). Intramuscular lesions formed via mineralization and eventual marrow-space development of a cartilaginous precursor, suggestive of an endochondral process (Supplemental Figure 3). Similar to the BMP-scaffold model, these mice demonstrated intramuscular damage on the basis of centralized myofiber nuclei (Figure 1B) and fibrosis (Figure 1C and Supplemental Figure 4) concurrent with, but separate from, the ectopic bone. Ad.cre/CTX did not cause ectopic bone or similar intramuscular damage (Figure 1, B and C) in littermate mice without the caAcvr $1^{\text {fl/fl }}$ transgene. Quantification of picrosirius red confirmed these findings (Figure 1D). Analysis of RNA expression extracted from injured muscle revealed increased expression of Colla1 transcripts, corroborating increased fibrotic deposition in $c a A c v$ f $^{f l / f l}$ mice when compared with wild type (Figure 1E).

We next quantified mesenchymal cells contributing to fibrosis using platelet-derived growth factor receptor $\alpha$ (PDGFRA) as an identifying marker. Fibrotic progenitor cells have previously been shown to express PDGFRA (33-35). Indeed, PDGFRA ${ }^{+}$cells were present, surrounding injured myofibers in both Ad.cre/CTX-treated wild-type and mutant mice (Figure 1F). FACS analysis confirmed that areas of overt fibrosis in Ad.cre/CTX-treated mutant mice had significantly more PDGFRA ${ }^{+}$cells (Figure $1, \mathrm{G}$ and $\mathrm{H}$ ). Importantly, myofiber injury and fibrosis preceded the osseous lesion, as indicated by histologic samples obtained 10 days after injury (Supplemental Figure 5, A-I). Taken together, these findings confirm the presence of a fibrotic lesion separate from the ectopic osseous lesion in the mouse model of FOP.

Rapamycin eliminates fibrosis associated with hyperactive BMP signaling. Although current therapeutic strategies for FOP focus on eliminating the osseous lesion, patients with FOP also have evidence of intramuscular inflammation on magnetic resonance imaging (MRI) (31). We have previously demonstrated that rapamycin eliminates ectopic bone in the Ad.cre/CTX-inducible mutant FOP mouse model. Therefore, we examined whether rapamycin similarly reduces or eliminates fibrosis in this model. Rapamycin markedly reduced myofiber injury (Figure 2A) and fibrosis (Figure 2, B and C) outside of the osseous lesions 20 days after injury. Furthermore, treatment diminished expression of Colla1 3 days after Ad.cre/CTX injury (Figure 2D). These findings were confirmed 10 days after injury, corresponding to the pre-osseous lesion in untreated mice (Supplemental Figure 6, A-F). Treatment with rapamycin also significantly reduced the presence of PDGFRA ${ }^{+}$cells in the hindlimb both 10 (Supplemental Figure 6, G-I) and 20 (Figure 2, E-G) days after injury. Taken together, these findings suggest that rapamycin eliminates both the ectopic osseous lesion and surrounding fibrosis experienced by patients with FOP.

Rapamycin preserves SMAD 1/5 signaling and osteogenic differentiation. Another subset of patients who may benefit from reduced muscle fibrosis and ectopic bone associated with BMP signaling are those with intramuscular BMP implants. However, in these patients, preservation of osteogenic differentiation conferred by BMP is critical. Interstitial mesenchymal cells isolated from the muscle of caAcvr1 $1^{f l f l}$ mice were transfected with Ad.cre or adenovirus control. As expected, Ad.cre-treated cells had significantly 
A
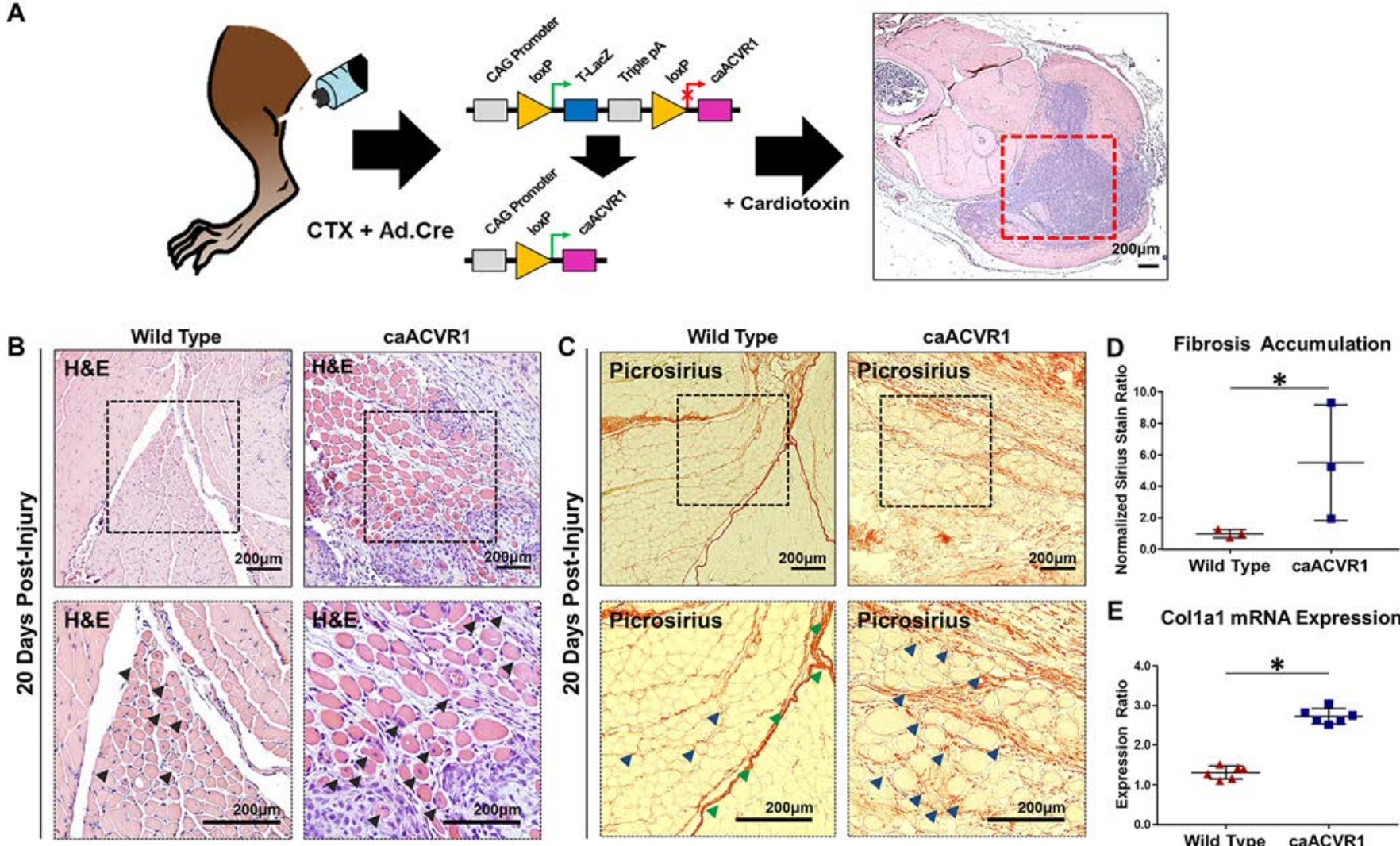

E Col1a1 mRNA Expression
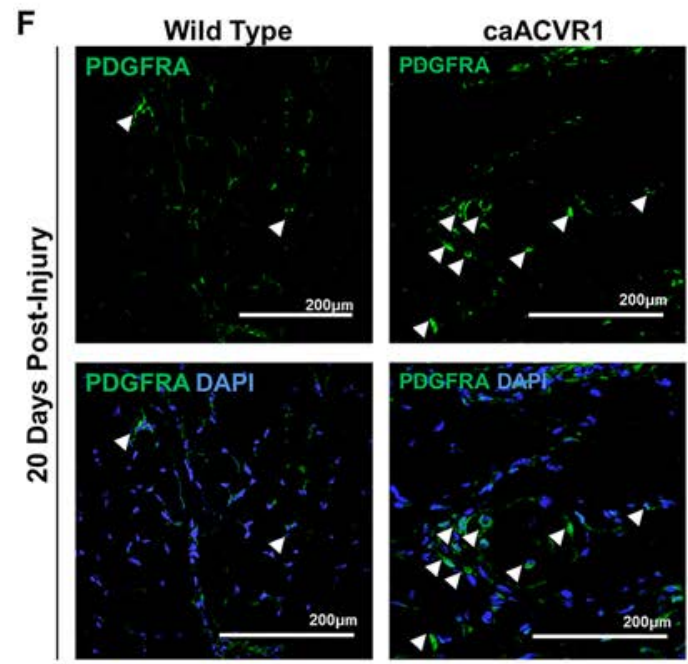

G

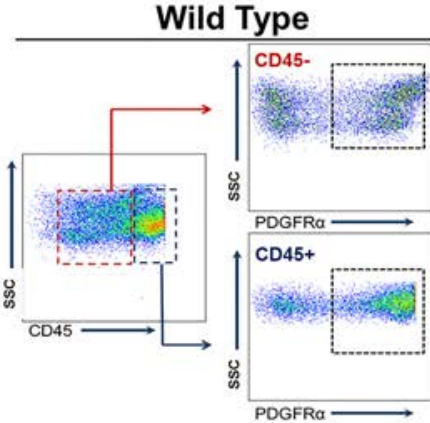

H

PDGFRA+; CD45+

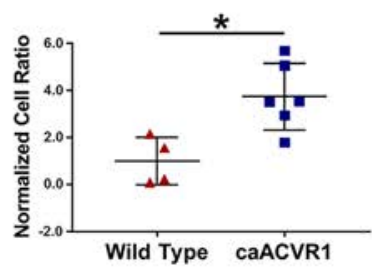

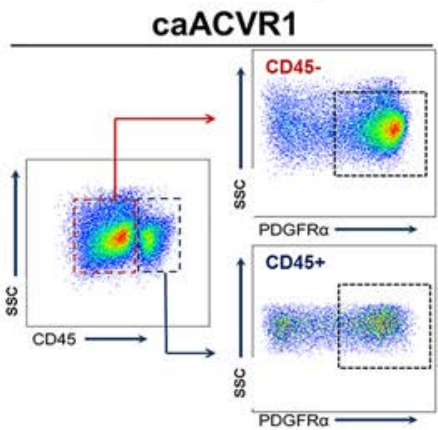

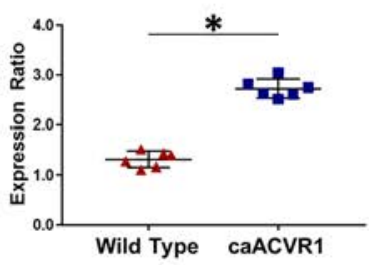

PDGFRA+; CD45-

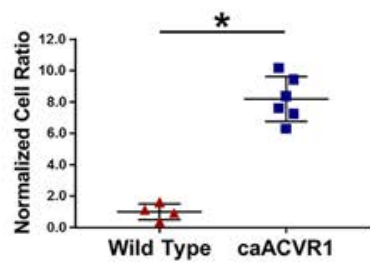

Figure 1. Myofiber injury and fibrosis surround the ectopic osseous lesion in a mouse model of hyperactive BMP signaling and local muscle injury. (A) Transgenic mouse model (caAcvr $\left.7^{f / f}\right)$ with activation of hyperactive BMP signaling after local adenoviral Cre (Ad.cre) injection. (B) Area of myofiber injury with centralized nuclei in wild-type mice 20 days after Ad.cre and cardiotoxin (CTX) injury (black arrowheads indicate myofibers with centralized nuclei). (C) Fibrosis corresponding with regions of myofiber injury in wild-type mice 20 days after Ad.cre/CTX injury (green arrowheads indicate fascial plane, blue arrowheads indicate fibrosis). (D) Increased fibrosis staining based on picrosirius red staining in caAcvr $7^{\text {fl/fl }}$ mice when compared with wild-type mice 20 days after Ad.cre/CTX injury (normalized ratio: 5.14 vs. 1.0, $P<0.05$, Student's 2 -tailed $t$ test, $n=3$ ). (E) Increased ratio of collagen 1 (Col1a1) mRNA expression in injured muscle harvested from untreated caAcvr1 $7^{f / f}$ mice when compared with wild-type mice (2.73 vs. 1.32, Student's 2-tailed $t$ test, $\left.n=6\right)$. (F) Representative immunostaining for platelet-derived growth factor receptor $\alpha$ (PDGFRA) in wild-type and caAcvr $7^{f / f / f}$ mice 20 days after Ad.cre/CTX injury (white arrowheads indicate PDGFRA ${ }^{+}$cells). (C) Gating strategy for FACS analysis to quantify PDGFRA ${ }^{+} C 45^{+}$(hematopoietic origin) and PDCFRA ${ }^{+}$CD45 ${ }^{-}$

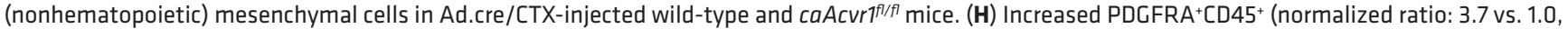

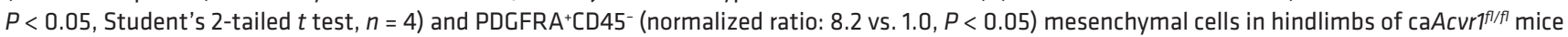
relative to wild-type mice 20 days after Ad.cre/CTX injury. SSC, side scatter. All scale bars: $200 \mu \mathrm{m} .{ }^{*} P<0.05$. 
A

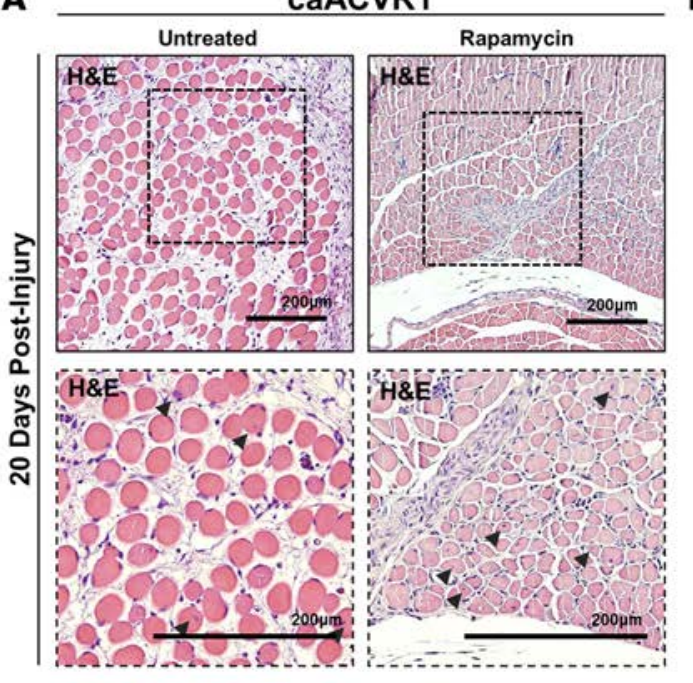

B
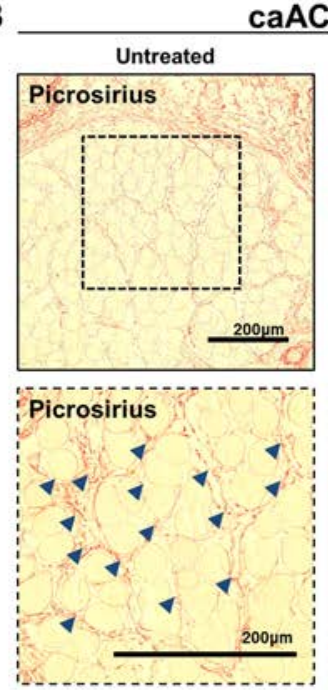

caACVR1

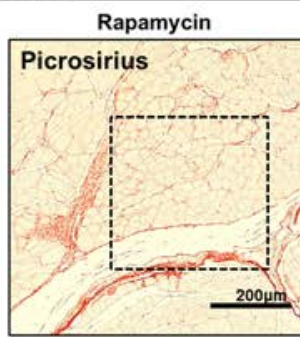

C

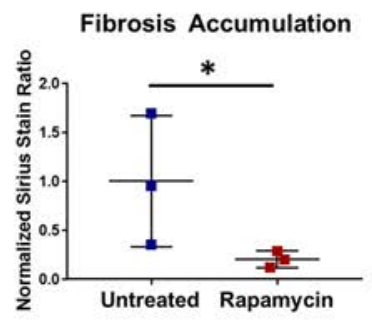

D Col1a1 mRNA Expression

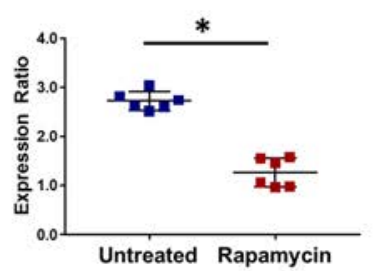

E

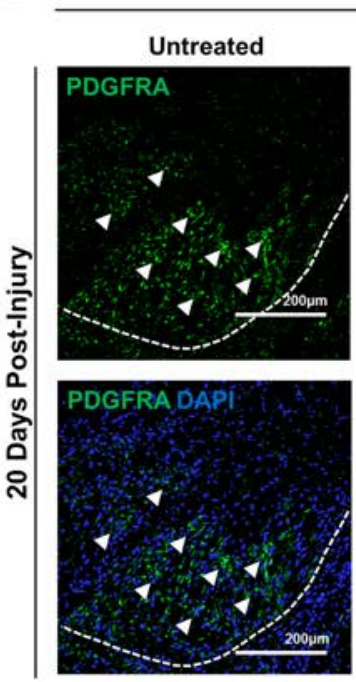

caACVR1
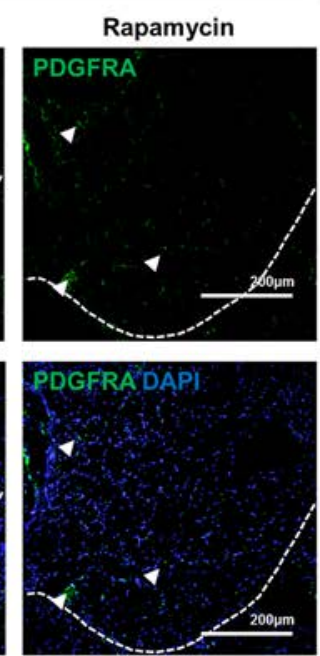

$\mathbf{F}$

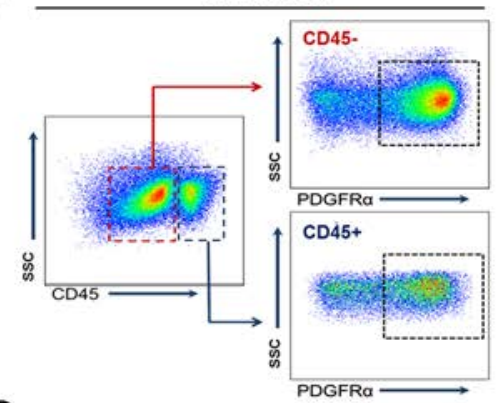

PDGFRA ; CD45+

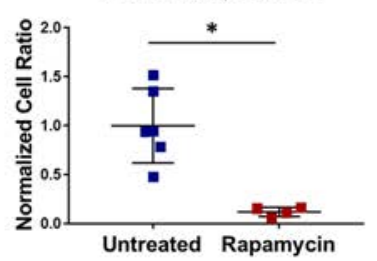

Rapamycin

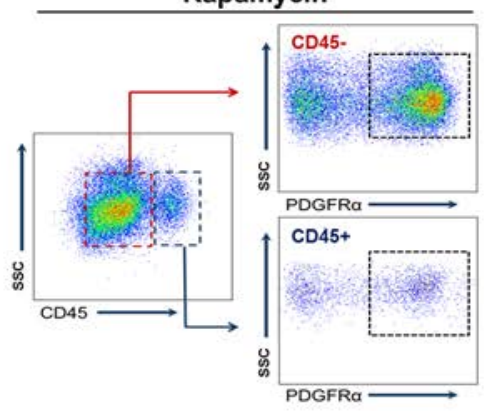

PDGFRA ; CD45-

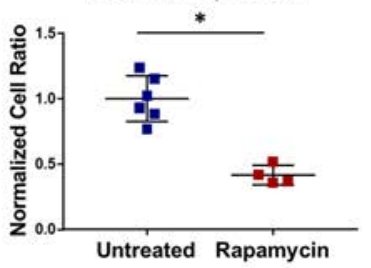

Figure 2. Rapamycin eliminates fibrosis associated with hyperactive BMP signaling. (A) Area of myofiber injury with centralized nuclei in untreated or rapamycin-treated caAcvr $7^{f / f l}$ mice 20 days after adenoviral Cre and cardiotoxin (Ad.cre/CTX) injury (black arrowheads indicate myofibers with centralized nuclei). (B) Fibrosis corresponding with regions of myofiber injury in untreated or rapamycin-treated caAcvrff/fl mice 20 days after Ad.cre/CTX injury (blue arrowheads indicate fibrosis). (C) Increased fibrosis based on picrosirius red staining in untreated caAcvr $7^{\text {fl/fl }}$ mice when compared with rapamycin-treated mice 20 days after Ad.cre/CTX injury (normalized ratio: 0.22 vs. 1.0, $P<0.05$, Student's 2-tailed $t$ test, $n=3$ ). (D) Increased ratio of collagen I (Col1a1) mRNA expression in injured muscle harvested from untreated caAcvr $1^{f / f l}$ mice when compared with rapamycin-treated mice (2.73 vs. 1.27, Student's 2-tailed $t$ test, $n=6$ ). (E) Representative immunostaining for platelet-derived growth factor receptor $\alpha$ (PDGFRA) in untreated and rapamycin-treated caAcvr $7^{f / f l}$ mice 20 days after Ad.cre/CTX injury (white arrowheads indicate PDGFRA ${ }^{+}$cells, white dashed line demarcates area of muscle injury). (F) Gating strategy for FACS analysis to quantify PDGFRA+CD45- (nonhematopoietic) and PDGFRA ${ }^{+}$CD45+ (hematopoietic origin) mesenchymal cells in untreated and rapamycin-treated Ad.cre/CTX-injured caAcvr $7^{f / f l}$ mice. (G) Increased PDGFRA+CD45- (normalized ratio: 0.42 vs. $1.0, P<0.05, n=6$ untreated, 4 rapamycin treated) and PDCFRA+CD45+ (normalized ratio: 0.12 vs. 1.0, $P<0.05, n=6$ untreated, 4 rapamycin treated) mesenchymal cells in hindlimbs from untreated caAcvr $7^{f / f l}$ mice relative to rapamycin-treated mice 20 days after Ad.cre/CTX injury. SSC, side scatter. All scale bars: $200 \mu \mathrm{m} .{ }^{*} P<0.05$.

increased in vitro osteogenic differentiation when compared with Ad.control-treated cells (Supplemental Figure 7). Furthermore, rapamycin increased osteogenic differentiation of Ad.cre-treated cells in vitro on the basis of mineral deposition, and immunoblot staining for p-SMAD 1/5 revealed no change in protein expression levels between treated and untreated groups (Figure 3, A and B). In vivo immunostaining showed preserved p-SMAD 1/5 in rapamycin-treated and untreated mutant mice 5 days after Ad.cre/ CTX injury (Figure 3C). These findings were confirmed on the basis of whole-tissue Western blot, with preserved p-SMAD 1/5 levels after Ad.cre/CTX injury and rapamycin treatment (Figure 3D). 


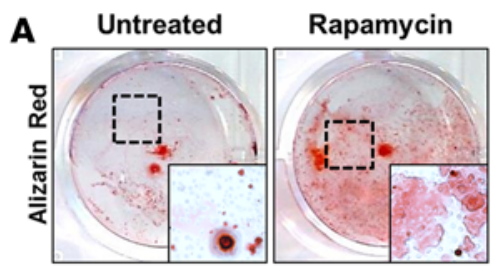

B

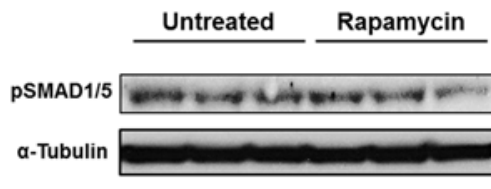

$\mathbf{C}$

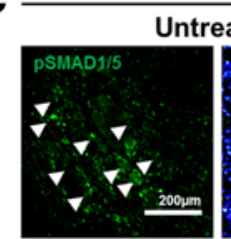

CaACVR1

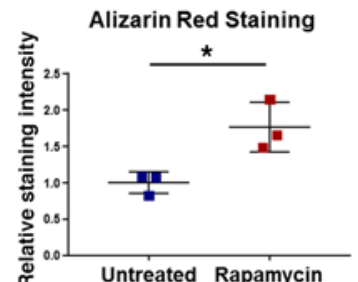

pSMAD 1/5
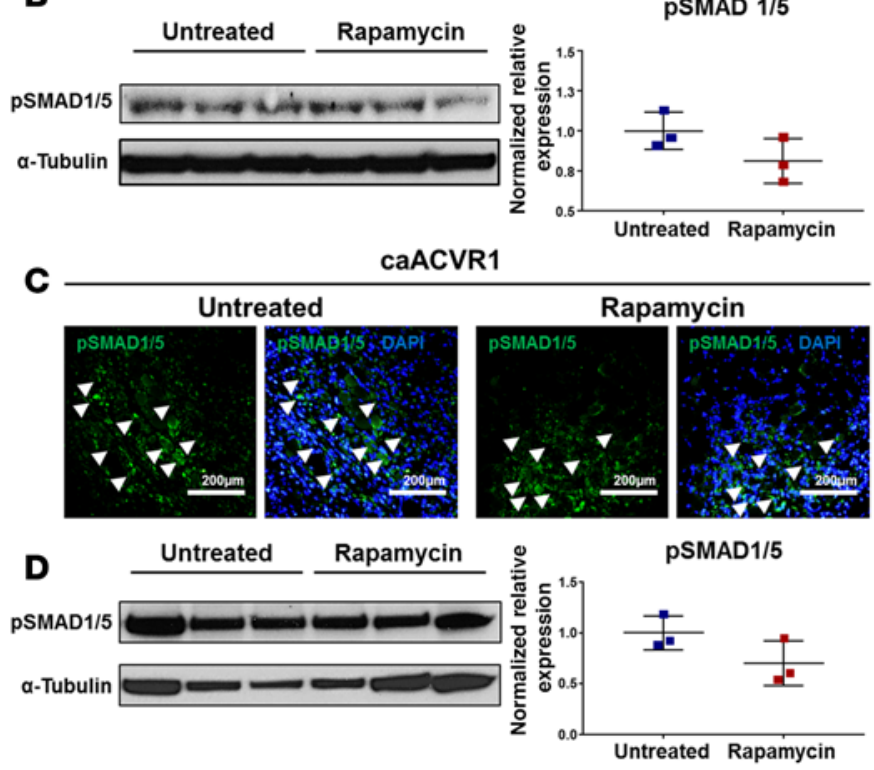

E

Cell Transplantation

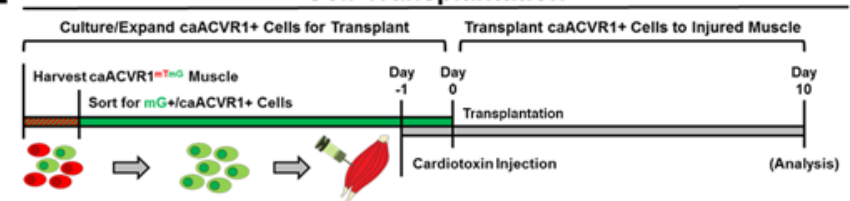

$\mathbf{F}$
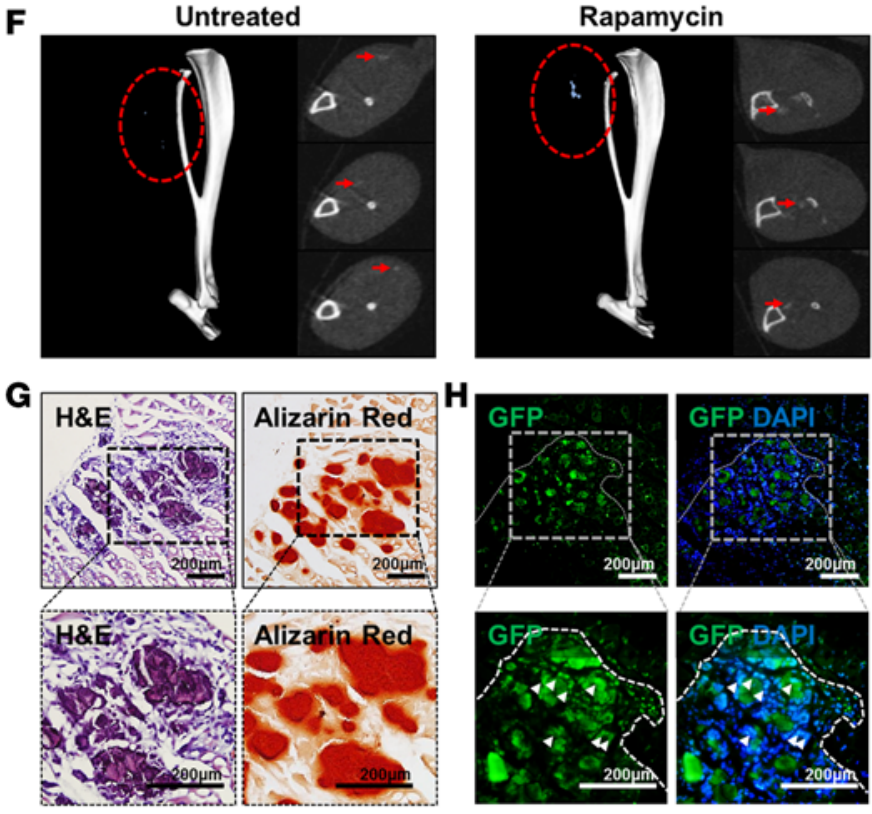

Figure 3. Rapamycin preserves SMAD 1/5 signaling and osteogenic differentiation. (A) Rapamycin significantly increased osteogenic differentiation of mesenchymal cells with hyperactive BMP signaling (normalized ratio: 1.77 vs. 1.0, Student's 2-tailed $t$ test, $n=3, P<0.05$ ). (B) Rapamycin preserves SMAD $1 / 5$ phosphorylation in mesenchymal cells with hyperactive BMP signaling in vitro (normalized ratio: 1.00 vs. 0.81 , Student's 2-tailed $t$ test, $n=3, P=0.15$, not significant). (C) Representative P-SMAD $1 / 5$ immunostaining in the hindlimbs of untreated and rapamycin-treated caAcvr ${ }^{f / f l}$ mice after adenoviral Cre and cardiotoxin (Ad.cre/CTX) injury (white arrowheads indicate p-SMAD 1/5 expression). (D) Whole-tissue immunoblot for $p$-SMAD 1/5 expression at the injury site of untreated and rapamycin-treated caAcvr $r^{f / / 7}$ mice after Ad.cre/CTX injury (normalized ratio: 1.00 vs. 0.70, Student's 2 -tailed $t$ test, $n=3, P=$ 0.13, not significant). (E) Experimental design for transplantation of mesenchymal cells with hyperactive BMP signaling. (F) Representative microCT reconstructions showing presence of ectopic bone in untreated and rapamycin-treated hindlimbs after transplantation of mesenchymal cells with hyperactive BMP signaling.(C) Representative H\&E and alizarin red staining of hindlimbs from rapamycin-treated mice after cell transplantation. (H) Representative fluorescence imaging showing expression of GFP from transplanted cells at sites of alizarin red staining; dotted border represents corresponding areas of muscle damage. All scale bars: $200 \mu \mathrm{m} .{ }^{*} P<0.05$.

Given that BMP signaling and osteogenic differentiation are preserved with rapamycin treatment, we hypothesized that the inhibitory effect of rapamycin may be due to reduced cell recruitment and exposure to Ad.cre at the injury site. Therefore, direct implantation of cells with hyperactive BMP signaling was performed to confirm their ability to form bone in vivo in rapamycin-treated mice. Wild-type mice received an injection of CTX followed by 50,000 GFP-labeled Ad.cre-treated caAcvr $1^{f / f l}$ cells 24 hours later to simulate cell recruitment (Figure 3E). Mice treated with rapamycin formed heterotopic ossification (HO), which was identifiable on microCT (Figure 3F). Histologic analysis showed that the presence of these cells corresponded with increased alizarin red staining, consistent with their contribution to ectopic bone (Figure $3, \mathrm{G}$ and $\mathrm{H}$ ). Taken together, these findings suggest that rapamycin does not reduce osteogenic potential conferred by BMP signaling.

Rapamycin after injury reveals improved myofiber size with hyperactive BMP signaling. Although the antiinflammatory and antifibrotic effects of rapamycin make this an enticing option to treat patients with muscle injury, studies have shown that mTOR inhibition actually has a detrimental effect on muscle size after injury $(2,30,36)$. Other studies have shown that BMP signaling has a positive effect on myofiber size and satellite cell expansion $(3,5)$. Therefore, we hypothesized that a dual therapy approach with rapamycin and elevated BMP signaling would simultaneously prevent fibrosis or ectopic bone formation, while improving myofiber size. First, we found that myofibers present in Ad.cre/CTX-injured caAcvr ${ }^{f l / f l}$ mice had increased 

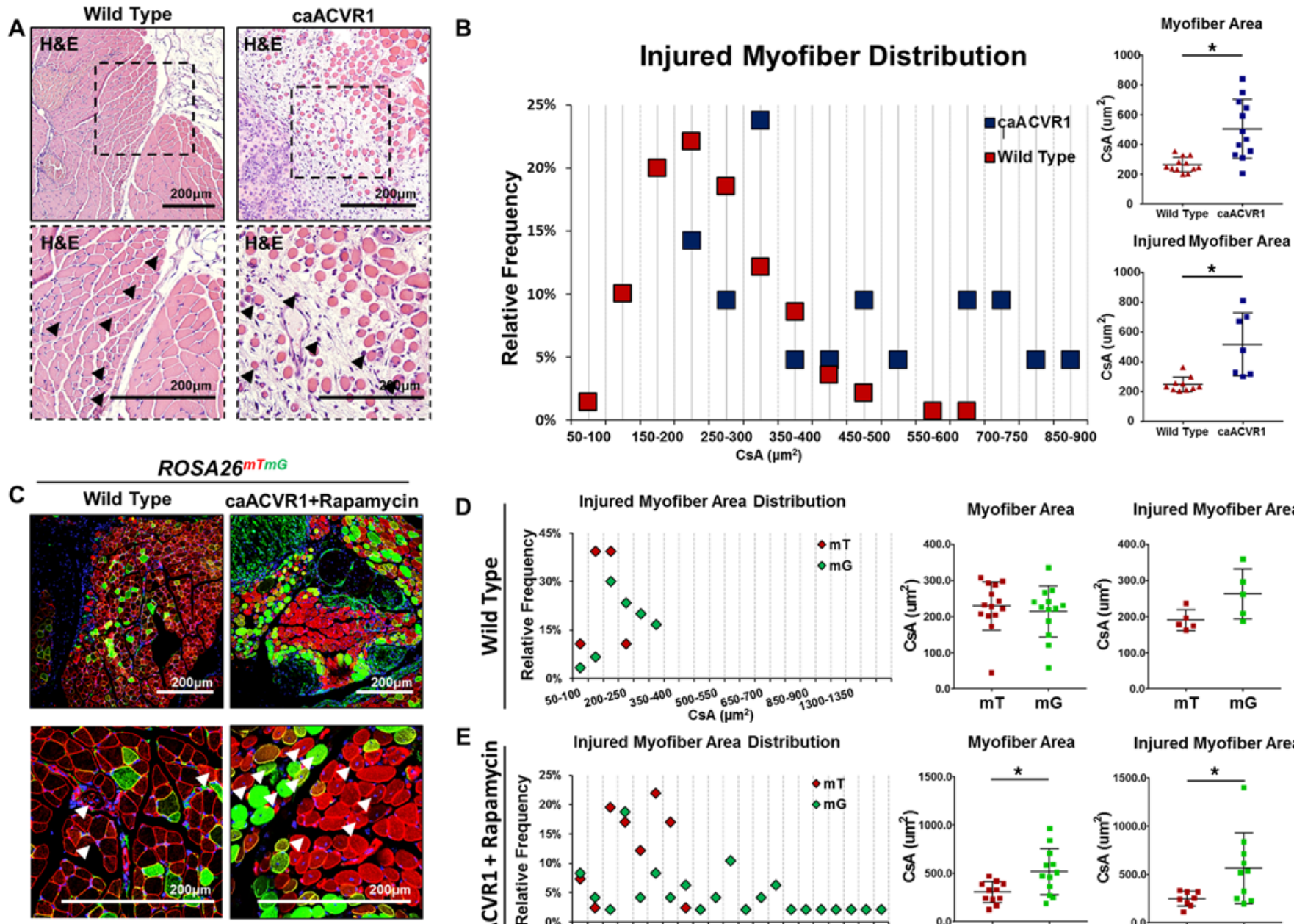

Injured Myofiber Area
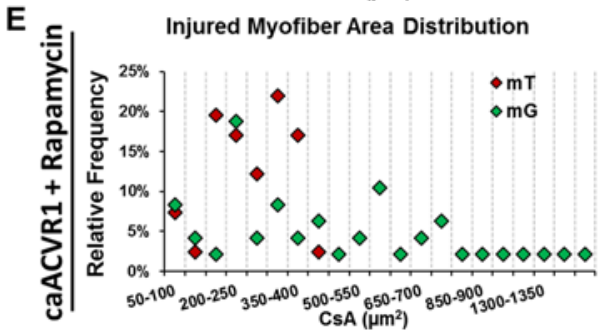

Myofiber Area
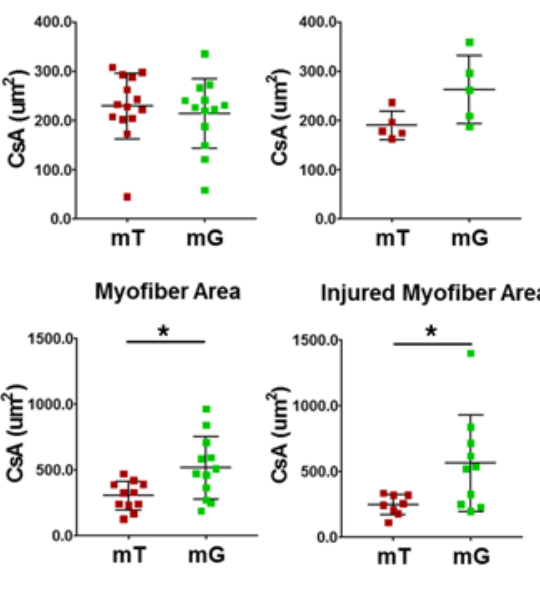

Injured Myofiber Area

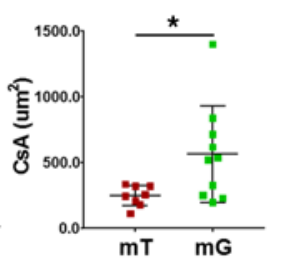

Figure 4. Rapamycin after injury reveals improved myofiber size with hyperactive BMP signaling. (A) H\&E showing areas of myofiber injury in wildtype and caAcvr $7^{f / f}$ mice 20 days after adenoviral Cre and cardiotoxin (Ad.cre/CTX) injury (black arrowheads indicate myofibers with centralized nuclei). (B) Distribution of myofiber cross-sectional areas showing significantly increased myofiber cross-sectional area in caAcvr $7^{f / f l}$ mice when compared with wild-type mice 20 days after Ad.cre/CTX injury (503 vs. $264 \mu \mathrm{m}^{2}$ for all myofibers, 551 vs. $251 \mu \mathrm{m}^{2}$ for centrally nucleated myofibers, Student's 2-tailed $t$ test, $n=4$ high-powered fields (HPFs) from 3 distinct biological samples, $P<0.05)$. (C) Representative fluorescence images of wild-type (ROSA26 $6^{m T m C}$ ) and mutant (caAcvr ${ }^{f / f l} R O S A 26^{m T m C}$ ) mice 20 days after Ad.cre/CTX injury and either no treatment (wild-type) or rapamycin treatment (mutant). (D) Distribution of green and red myofiber cross-sectional area $\left(\mu \mathrm{m}^{2}\right)$ in wild-type ROSA26 ${ }^{\mathrm{mTm} G}$ mice 20 days after Ad.cre/CTX injury showing absence of statistically significant or substantial difference in cross-sectional area (227 vs. $243 \mu \mathrm{m}^{2}$, Student's 2-tailed $t$ test, $n=4 \mathrm{HPFs}$ from 3 distinct biological samples, $P$ = not significant). (E) Distribution of green and red myofiber cross-sectional area $\left(\mu \mathrm{m}^{2}\right)$ in caAcvr $7^{f / f l} R O S A 26^{m T m G}$ mice 20 days after Ad.cre/ CTX injury showing significantly increased green myofiber cross-sectional area (519 vs. $305 \mu \mathrm{m}^{2}$ for all myofibers, 551 vs. $251 \mu \mathrm{m}^{2}$ for injured myofibers, Student's 2-tailed $t$ test, $n>7$ HPFs from 3 distinct biological samples, $P<0.05)$. ${ }^{*} P<0.05$. All scale bars: $200 \mu \mathrm{m}$. CsA, cross-sectional area; mT, membrane-bound RFP; $\mathrm{mG}$, membrane-bound GFP.

cross-sectional area when compared with the myofibers present in Ad.cre/CTX-injured wild-type mice (503 vs. $264 \mu \mathrm{m}^{2}$ ) (Figure 4, A and B). However, these findings are confounded by the presence of marked fibrosis and muscle damage in $c a A c v r 1^{1 / f l}$ mice. To further evaluate whether BMP signaling can improve myofiber size in the absence of pervasive fibrosis, wild-type reporter mice $\left(R O S A 26^{\mathrm{mTm} G}\right)$ and mutant reporter mice ( $c a A c v r 1^{1 / f l} R O S A 26^{m T m G}$ ) were injured with Ad.cre/CTX. In this model, those cells that have been transformed by Ad.cre, or are descended from transformed cells, will appear green while those that have not will appear red (Figure 4C). This serves as an indicator for hyperactive BMP signaling, and the internal comparison between myofibers provides an additional control against local tissue changes induced by the Ad.cre/CTX injury. This strategy of cell labeling as a proxy for upregulated BMP signaling in the mutant reporter was confirmed on the basis of increased in vitro osteogenic differentiation observed among mesenchymal cells derived from floxed mice treated with Ad.cre when compared with cells treated with control 


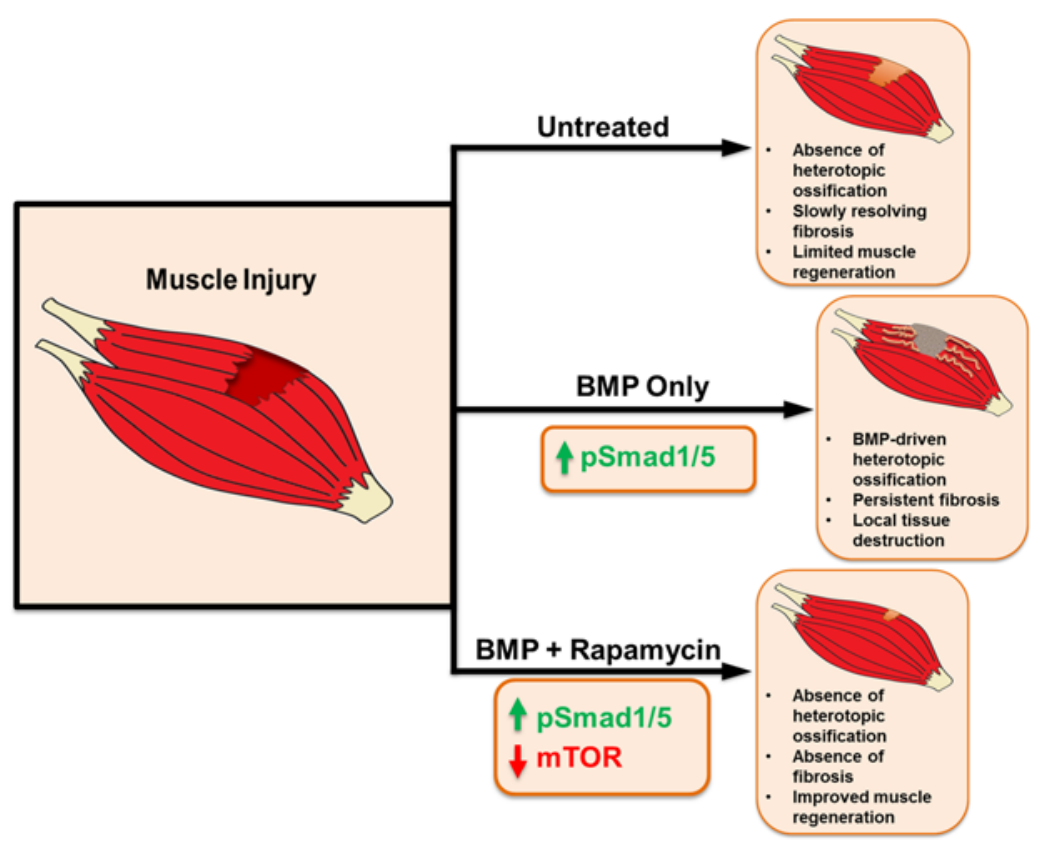

Figure 5. Schematic showing effects of rapamycin and BMP signaling after muscle injury.

adenovirus (Supplemental Figure 7). Green and red myofibers from wild-type reporter mice 20 days after Ad.cre/CTX injury showed similar cross-sectional areas (226 vs. $243 \mu \mathrm{m}^{2}, P=$ not significant) (Figure $4 \mathrm{D})$. However, green myofibers from rapamycin-treated caAcvr $1^{\beta / l} R O S A 26^{m T m G}$ mice 20 days after Ad.cre/ CTX injury had over $70 \%$ increased cross-sectional area when compared with red myofibers (519 vs. 305 $\mu \mathrm{m}^{2}, P<0.05$ ) (Figure 4E). Analysis of myofiber distribution showed a shift towards increased green myofiber cross-sectional area when compared with red myofibers (Figure 4E).

\section{Discussion}

The findings from this study indicate that rapamycin can eliminate fibrosis associated with muscle injury in the setting of increased BMP signaling, as is observed in patients with FOP. Therefore, in addition to pre-

venting ectopic bone formation (29), rapamycin may be able to alleviate additional muscle pathology in these patients. Interestingly, these patients do exhibit evidence of muscle inflammation that precedes ectopic ossification and therefore may be preventable with rapamycin (31).

Consistent with other studies, our findings also indicate that rapamycin preserves BMP signaling and does not reduce osteogenic differentiation (37). Therefore, rapamycin may be a useful adjunct by reducing inflammation but preserving osteogenesis in patients who have rhBMP2 implants to improve bone healing. Rapamycin appears to reduce the presence of mesenchymal cells $\left(\mathrm{PDGFRA}^{+}\right)$at the injury site, which may influence both the fibrotic scar and heterotopic bone together.

Finally, these findings demonstrate that in the setting of muscle injury, concomitant rapamycin with increased BMP signaling can alleviate fibrosis and simultaneously increase myofiber size. Effectively, the adverse effect of each therapy is offset by the other. Rapamycin has been shown to reduce fibrosis in several tissue types (21-28). However, in the setting of muscle injury, this comes at a cost, as mTOR/AKT signaling regulates myofiber size $(2,30)$. Previous studies that shown that, while the early phases of muscle regeneration proceed with rapamycin, myofiber growth is halted (38). Separately, BMP through type I receptor activity has been shown to positively regulate muscle size (3-5). Previous studies have been unable to demonstrate a positive effect of BMP signaling on myofiber size after muscle injury, primarily owing to the robust formation of ectopic bone and muscle destruction that occur with local BMP delivery (6-10). Our findings suggest that a combined therapeutic approach of rapamycin with increased BMP signaling can reduce or eliminate fibrosis while improving myofiber size.

Previous studies have shown that BMP signaling improves myofiber size through SMAD 1/5-mediated activation of the mTOR/AKT pathway (37). Despite inhibition of mTOR/AKT using rapamycin, we show that increased BMP signaling increases myofiber size, suggesting that SMAD 1/5-mediated increases in myofiber size may also occur independently of mTOR/AKT. These findings should be interpreted with caution, however, as rapamycin may not have completely eliminated mTOR/AKT signaling, especially in myofibers with elevated BMP signaling. We also found that rapamycin increases osteogenic signaling in vitro and in vivo, consistent with other reports - these findings indicate that mTOR inhibition reduces fibrosis and $\mathrm{HO}$ through a SMAD 1/5-independent mechanism. Ultimately, these findings suggest that increased BMP signaling alone is not responsible for muscle damage in the setting of injury, but that concomitant activation of the mTOR pathway leads to fibrosis and ectopic bone formation.

To study the effect of BMP signaling on myofiber size, we first compared the size of myofibers present in wild-type and caAcvr1 ${ }^{f l f l}$ mice 20 days after Ad.cre/CTX injury. Those myofibers that were present in the mutant mice had increased cross-sectional area when compared with wild-type mice. However, there were fewer myofibers overall at sites of injury due to the extensive damage. Having identified rapamycin 
as an inhibitor of fibrosis in the setting of increased BMP signaling, we next treated caAcvr $1^{f l / f l}$ ROSA26 $6^{m T m G}$ mice with rapamycin after Ad.cre/CTX injury to specifically evaluate whether the increased myofiber size associated with BMP is preserved. By comparing red and green myofibers within the same reporter mouse, we were able to control for local tissue changes caused by the Ad.cre/CTX injection. Because the red and green myofibers are in the same mouse at the same relative location within high-power fields (HPFs), we make the assumption that these myofibers are exposed to similar local conditions. That the reporter system correctly identifies green cells as having upregulated BMP signaling was verified using an assay to confirm that green cells have increased osteogenic differentiation when compared with red cells from these mice. In this comparison, green myofibers appeared to have significantly and substantially increased cross-sectional area when compared with red myofibers. We also evaluated wild-type reporter mice that had received Ad.cre/CTX and noted that green and red myofibers had similar sizes, suggesting that BMP signaling was responsible for the observed effect in mutant reporter mice. These wild-type reporter mice did not receive rapamycin because they did not show evidence of long-standing fibrosis. Overall, our findings indicate that BMP signaling may alleviate myofiber atrophy associated with injury and with rapamycin treatment, while rapamycin can eliminate fibrosis associated with injury and ectopic bone associated with BMP.

Our study has some important limitations. To study the effect of BMP signaling on myofiber injury, we used a mutant reporter mouse with the floxed caAcvr1 transgene. We felt this was an appropriate system to use for several reasons including the ability to make direct comparisons within the same mouse using the reporter label, and because constitutively active ACVR1 increases phosphorylated SMAD 1/5 (7), a critical downstream mediator of BMP signaling. Furthermore, implantation of the BMP ossicle demonstrated a similar formation of ectopic bone and surrounding myofiber injury and fibrosis as observed in Ad.cre/ CTX-treated mutant mice. An additional limitation is that we were unable to assess functional metrics in these mice. This is because Ad.cre/CTX injury induces a destructive phenotype in mutant mice and therefore leads to severe functional impairment including joint contracture (7). Therefore, it is not reasonable to directly compare rapamycin-treated and untreated mice, although visually rapamycin-treated mice appear to have unperturbed gait. It may also not be appropriate to compare the functional metrics of rapamycin-treated and untreated wild-type mice after Ad.cre/CTX treatment, owing to the overall absence of long-standing fibrosis in these mice even without rapamycin. However, previous studies have shown that increased myofiber size correlates with increased functional capacity (3). Finally, we are unable to determine whether increased myofiber size comes at the expense of satellite cell populations in our model. Previous studies, however, have indicated that BMP signaling actually prevents premature satellite cell differentiation, suggesting that this is not the case in our model (5).

Overall, our study evaluates a synergistic approach to reducing or eliminating fibrosis after muscle injury while improving myofiber size through combined rapamycin treatment and hyperactive BMP signaling (Figure 5). Clinically, it is feasible to systemically deliver rapamycin and locally inject BMP at sites of injury. An approach in which 2 therapies offset each other's adverse effects is insightful into a broad therapeutic strategy.

\section{Methods}

Transgenic animals. caAcvr1 $1^{f / f l}$ mice were donated by Yuji Mishina. caAcvr $1^{f / f l} R O S A 26^{m T m G}$ mice were bred by crossing caAcvrlf $f^{\prime f l}$ mice with ROSA26 $6^{m T m G}$. Tail genomic DNA was used for genotyping to confirm mutant status for each generation of pups.

Induction of fibrosis. Transgenic and wild-type control mice were induced at similar ages with a combined injection of Ad.cre $\left(0.7 \times 10^{9} \mathrm{PFU} /\right.$ injection site) and CTX (900 ng/site) (Latoxan) in a total volume of $10 \mu \mathrm{l}$ to the hindlimb gastrocnemius muscle. Mice receiving injections were further subdivided into treatment and nontreatment groups on the basis of further therapeutic interventions (see below). Following injection, mice from each group were maintained for 5, 10, and 20 days after injury until harvest for further analysis.

Rapamycin treatment. Mice assigned to treatment groups received Ad.cre and CTX as described above followed by daily intraperitoneal injections of rapamycin $(5 \mathrm{mg} / \mathrm{kg})$ (MedKoo) up to the day of harvest. Nontreatment controls received daily intraperitoneal saline as vehicle control.

Cell injection. A second cohort of mice received an injection of CTX into the hindlimb musculature followed by direct injection of transformed caAcvr1 cells (50,000 cells in $25 \mu 1$ PBS) directly into the injury site 24 hours later. Mice were treated with rapamycin or vehicle control as above and were subsequently sacrificed for further analysis. 
Histologic processing. At 10 or 20 days after injury animals were euthanized for histology. At the time of euthanization, the hindlimb with musculature was sharply dissected. Samples were fixed overnight in $10 \%$ formalin at $4^{\circ} \mathrm{C}$ and then washed in PBS and transferred to $19 \%$ EDTA solution for 28 days at $4^{\circ} \mathrm{C}$ to decalcify osseous tissues. After decalcification, samples were washed and transferred to $15 \%$ sucrose for 24 hours. Following cryopreservation, samples were wiped clean of residual sucrose and cryoembedded in OCT (Tissue-Tek). All cryoembedded samples were cut to a thickness of 5 to $7 \mu \mathrm{m}$ and mounted on positively charged slides for further histologic analysis.

Histologic analysis. Cryopreserved slides were stained with H\&E to assess baseline morphology, and picrosirius red and Masson's trichrome to assess collagen content. All picrosirius red staining was conducted under standardized conditions for quantification. Briefly, 3 hindlimb specimens were collected from groups 10 and 20 days after injury. Three slides at regular intervals were stained per hindlimb specimen. Images were obtained at $\times 20$ magnification for areas of interest defined by the inclusion of both (a) areas of damage and (b) surrounding muscle tissue. Staining intensity for picrosirius red was determined using Adobe Photoshop, using the pixel ratio of red collagen tissue to yellow tissue using RBG signature. Quantification was conducted by 3 independent, blinded participants.

Immunohistochemistry. Immunohistochemical staining was performed with the following antibodies at a 1:50 dilution: anti-PDGFRA (Santa Cruz Biotechnology, C-20) and anti-p-SMAD 1/5 (Santa Cruz Biotechnology, sc-12353). Secondary antibodies were applied that were directed against the host animal of the primary antibody. Nuclear counterstain was achieved with DNA dye DAPI (Thermo Scientific) and samples were mounted with aqueous mounting media (Sigma-Aldrich). Four hindlimb specimens were taken from mice in respective groups 10 and 20 days after injury. Slides for each specimen were stained for PDGFRA. Images were obtained at $\times 20$ magnification for areas of interest defined by the inclusion of both (a) areas of damage and (b) surrounding muscle tissue. Eight HPFs were randomly selected for each stain. Immunofluorescence staining was regarded as positive when signal was immediately proximal to $\mathrm{DAPI}^{+}$staining. Raw numbers of positive marker signaling and all $\mathrm{DAPI}^{+}$cells were counted for each HPF selected. Relative frequency was calculated as the average of the ratio of PDG$\mathrm{FRA}^{+}$to $\mathrm{DAPI}^{+}$cells for each slide.

Quantification of muscle regeneration. Three hindlimb specimens were collected from the respective groups. For each hindlimb, 4 HPFs were randomly selected using a random number generator to quantify mean cross-sectional area of $\mathrm{GFP}^{+}$and red fluorescent protein ${ }^{+}\left(\mathrm{RFP}^{+}\right)$myofibers. ImageJ $(\mathrm{NIH})$ was used to quantify total cross-sectional area of all $\mathrm{GFP}^{+}$or $\mathrm{RFP}^{+}$cells. The mean was determined by dividing the cross-sectional areas by the total number of cells within the HPF.

Cell culture. Fibroblast cells were isolated from the hindlimb musculature of caAcvr1 ${ }^{f / f l}$ ROSA26 $6^{m T m C}$ mutants. Cultures of at least 3 independent biological specimens were maintained for in vitro experiments. Cells were transfected with Ad.cre or Ad.control and sorted by FACS to purify populations of green cells from the Ad.cre-transfected wells and red cells from the Ad.control-transfected wells

Osteogenic differentiation. Transfected and untransfected populations as described above were seeded in triplicate onto 6-well plates at a density of 100,000 cells/well and onto a 12-well plate at a density of 35,000 cells/well. After 24 hours, media was changed to osteogenic differentiation media (ODM) containing DMEM, 10\% fetal bovine serum, $100 \mathrm{~g} / \mathrm{ml}$ ascorbic acid, $10 \mathrm{mM} \beta$-glycerophosphate, and $100 \mathrm{IU} / \mathrm{ml}$ penicillin/streptomycin. Early osteogenic differentiation was assessed by alkaline phosphatase (ALP) stain and quantification of ALP enzymatic activity after 7 days in ODM. Production of osteoid was measured by alizarin red staining.

In vitro rapamycin treatment. Transfected and untransfected cell populations were isolated for osteogenic differentiation as described above. These populations were then further subdivided into treated and untreated samples. All treated samples received $100 \mathrm{nM}$ rapamycin added to their normal cell growth media.

Flow cytometry. Skeletal muscle of Ad.cre/CTX-injured hindlimbs from wild-type and caAcvr1 ${ }^{\text {fl/fl }}$ mice with and without rapamycin treatment were harvested 20 days following initial injury under sterile conditions ( $n=4-6$ mice for each experimental group). Muscle tissue was mechanically disrupted and digested in a solution of collagenase type I $\left(0.5 \%\right.$ in HBSS) for 1 hour at $37^{\circ} \mathrm{C}$. Tissue homogenate was then passed through a $70-\mu \mathrm{m}$ strainer, and stained on ice in FACS buffer $(0.5 \%$ BSA, 2 mM EDTA in Dulbecco's PBS) with anti-CD140a (eBioscience, 12-1401) and anti-mouse CD45 APC (eBioscience, 30-F11) for 1 hour. Data were acquired on a FACS ARIA II instrument (BD Biosciences) and analyzed using FlowJo software. 
Quantitative PCR. Total RNA was extracted from hindlimb muscle 3 days following Ad.cre/CTX injury using an RNAeasy Kit (Qiagen) per the manufacturer's specifications. Reverse transcription was performed with $1 \mu \mathrm{g}$ RNA using a cDNA Reverse Transcription Kit (Applied Biosystems). Quantitative real-time PCR was carried out using the Applied Biosystems Prism 7900HT Sequence Detection System and SYBR Green PCR Master Mix (Applied Biosystems). The PCR protocol included a $95^{\circ} \mathrm{C}$ denaturation (20 seconds), annealing (20 seconds), and $72^{\circ} \mathrm{C}$ extension (30 seconds). Detection of fluorescent product was carried out at the end of the $72^{\circ} \mathrm{C}$ extension period. PCR products underwent melting curve analysis, and the data were analyzed and quantified with the relative expression software tool (REST; http://www.gene-quantification.com/rest.html). Dynamic tube normalization and noise slope correction was used to remove background fluorescence. Each sample was tested in triplicate and repeated for 3 independent biologic preparations. Primers used for Colla1 were (5'-3') GCCAAGGCAACAGTCGCT and CTTGGTGGTTTTGTATTCGATGAC.

MicroCT. In vivo development of HO was assessed with microCT scans 20 days after injury (GE Healthcare Biosciences, using $80 \mathrm{kVp}, 80 \mathrm{~mA}$, and 1,100 ms exposure). Images were reconstructed and $\mathrm{HO}$ volume formation was analyzed using a calibrated imaging protocol as previously described (39).

Microscopy. All tissue sections and fluorescently stained samples were imaged using either (a) a Zeiss LSM 510-META Laser Scanning Confocal Microscope equipped with 4 lasers: coherent Enterprise laser (UV/DAPI), argon laser (FITC/GFP), helium neon 1 laser (AF594, RFP), and helium neon 2 laser (AF647); or (b) an Olympus BX-51 upright light microscope equipped with standard DAPI, 488, and TRITC reflector cubes attached to an Olympus DP-70 high-resolution digital camera. H\&E- and picrosirius-stained sections were imaged at $\times 10$ and $\times 20$ magnification. Immunofluorescence images were taken at either $\times 20$ or $\times 40$ magnification. Scale bars were placed for all images with a standard $200-\mu \mathrm{m}$ diameter. Sites were imaged in all channels and overlaid in DPView (Olympus America) before examination in Adobe Photoshop or ImageJ.

Statistics. All statistical analyses were performed using SPSS v20 (IBM). All experiments were performed with $n \geq 3$ using 2-way ANOVA or Student's 2-tailed $t$ test. Means and SDs were calculated from numerical data, as presented in the text, figures, and figure legends. All graphs are presented as dot plots, with means indicated by horizontal bars. Error bars represent $1 \mathrm{SD}$. Asterisks are representative of statistical significance. Statistical significance was determined as a $P$ value less than 0.05 .

Study approval and animal use. Animal procedures were conducted in accordance with guidelines from the NIH Guide for the Use and Care of Laboratory Animals: Eighth Edition, and approved by the Institutional Animal Care and University Committee on the Use and Care of Animals of the University of Michigan (UCUCA PRO0005909). All animals were housed in UCUCA-supervised facilities, not to exceed 3 mice housed per cage at $72^{\circ} \mathrm{F} \pm 4^{\circ} \mathrm{F}$, receiving 12 hours of light exposure each day, with no diet restrictions. Young (3- to 4-week-old) mice were used for all experiments. Wild-type C57BL/6 mice were used for all

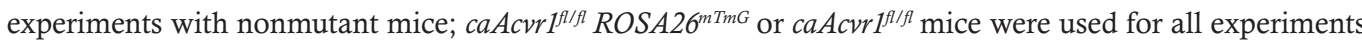
with mutant mice.

\section{Author contributions}

SA, DC, and BL designed the experiments. SA, DC, and MTC performed all mouse injuries. SA, DC, S. Loder, JL, JP, MTC, S. Li, KR, JH, JD, CB, MC, and HHS, collected data. YM provided transgenic mice and helped with study design and analysis. SA, S. Loder, HHS, YM, and BL reviewed data and edited the manuscript. SA, DC, and BL drafted the manuscript.

\section{Acknowledgments}

The authors would like to thank the staff at the University of Michigan Microscopy and Imaging Laboratory (MIL), and Center for Molecular Imaging (CMI) cores, particularly Amanda Fair. SA was supported by a Coller Surgical Society Research Fellowship, the NIH Loan Repayment Program, NIH grant F32AR06649901A1, and the Plastic Surgery Foundation. SL was supported by the Howard Hughes Medical Institute (HHMI) Medical Fellows Program. KR was supported by NIH grant F32 AR068902. BL was supported by funding from the NIH/National Institute of General Medical Sciences grant K08GM109105-0, an American Association of Plastic Surgery Academic Scholarship and joint PSF Pilot Award, an Association for Academic Surgery Roslyn Award, an American Association for the Surgery of Trauma Research \& Education Foundation Scholarship, and the International FOP Association. 
Address correspondence to: Benjamin Levi, 1150 West Medical Center Drive, Ann Arbor, Michigan 48109, USA. Phone: 734.936.0034; E-mail: blevi@umich.edu.

1. Liu X, Joshi S, Ravishankar B, Laron D, Kim HT, Feeley BT. Bone morphogenetic protein signaling in rotator cuff muscle atrophy and fatty infiltration. Muscles Ligaments Tendons J. 2015;5(2):113-119.

2. Bodine SC, et al. Akt/mTOR pathway is a crucial regulator of skeletal muscle hypertrophy and can prevent muscle atrophy in vivo. Nat Cell Biol. 2001;3(11):1014-1019.

3. Sartori R, et al. BMP signaling controls muscle mass. Nat Genet. 2013;45(11):1309-1318.

4. Winbanks CE, et al. The bone morphogenetic protein axis is a positive regulator of skeletal muscle mass. $J$ Cell Biol. 2013;203(2):345-357.

5. Ono Y, Calhabeu F, Morgan JE, Katagiri T, Amthor H, Zammit PS. BMP signalling permits population expansion by preventing premature myogenic differentiation in muscle satellite cells. Cell Death Differ. 2011;18(2):222-234.

6. Shimono K, et al. Potent inhibition of heterotopic ossification by nuclear retinoic acid receptor- $\gamma$ agonists. Nat Med. 2011;17(4):454-460

7. Yu PB, et al. BMP type I receptor inhibition reduces heterotopic [corrected] ossification. Nat Med. 2008;14(12):1363-1369.

8. Peterson JR, et al. Treatment of heterotopic ossification through remote ATP hydrolysis. Sci Transl Med. 2014;6(255):255ra132

9. Agarwal S, et al. BMP signaling mediated by constitutively active activin type 1 receptor (ACVR1) results in ectopic bone formation localized to distal extremity joints. Dev Biol. 2015;400(2):202-209.

10. McKay WF, Peckham SM, Badura JM. A comprehensive clinical review of recombinant human bone morphogenetic protein-2 (INFUSE Bone Graft). Int Orthop. 2007;31(6):729-734.

11. Skovrlj B, Marquez-Lara A, Guzman JZ, Qureshi SA. A review of the current published spinal literature regarding bone morphogenetic protein-2: an insight into potential bias. Curr Rev Musculoskelet Med. 2014;7(3):182-188.

12. Adams CL, Ogden K, Robertson IK, Broadhurst S, Edis D. Effectiveness and safety of recombinant human bone morphogenetic protein-2 versus local bone graft in primary lumbar interbody fusions. Spine. 2014;39(2):164-171.

13. Simmonds MC, et al. Safety and effectiveness of recombinant human bone morphogenetic protein-2 for spinal fusion: a metaanalysis of individual-participant data. Ann Intern Med. 2013;158(12):877-889.

14. Garrett MP, Kakarla UK, Porter RW, Sonntag VK. Formation of painful seroma and edema after the use of recombinant human bone morphogenetic protein-2 in posterolateral lumbar spine fusions. Neurosurgery. 2010;66(6):1044-9; discussion 1049.

15. Glassman SD, et al. RhBMP-2 versus iliac crest bone graft for lumbar spine fusion: a randomized, controlled trial in patients over sixty years of age. Spine. 2008;33(26):2843-2849.

16. Villavicencio AT, Burneikiene S, Nelson EL, Bulsara KR, Favors M, Thramann J. Safety of transforaminal lumbar interbody fusion and intervertebral recombinant human bone morphogenetic protein-2. J Neurosurg Spine. 2005;3(6):436-443.

17. Baskin DS, Ryan P, Sonntag V, Westmark R, Widmayer MA. A prospective, randomized, controlled cervical fusion study using recombinant human bone morphogenetic protein-2 with the CORNERSTONE-SR allograft ring and the ATLANTIS anterior cervical plate. Spine. 2003;28(12):1219-24; discussion 1225.

18. Woo EJ. Adverse events after recombinant human BMP2 in nonspinal orthopaedic procedures. Clin Orthop Relat Res. 2013;471(5):1707-1711.

19. Shore EM, et al. A recurrent mutation in the BMP type I receptor ACVR1 causes inherited and sporadic fibrodysplasia ossificans progressiva. Nat Genet. 2006;38(5):525-527.

20. Fukuda T, et al. Constitutively activated ALK2 and increased SMAD1/5 cooperatively induce bone morphogenetic protein signaling in fibrodysplasia ossificans progressiva. J Biol Chem. 2009;284(11):7149-7156.

21. Hillel AT, Gelbard A. Unleashing rapamycin in fibrosis. Oncotarget. 2015;6(18):15722-15723.

22. Shao X, et al. Effects of rapamycin against paraquat-induced pulmonary fibrosis in mice. J Zhejiang Univ Sci B. 2015;16(1):52-61.

23. Liu CF, Liu H, Fang Y, Jiang SH, Zhu JM, Ding XQ. Rapamycin reduces renal hypoxia, interstitial inflammation and fibrosis in a rat model of unilateral ureteral obstruction. Clin Invest Med. 2014;37(3):E142.

24. Jin X, Dai H, Ding K, Xu X, Pang B, Wang C. Rapamycin attenuates bleomycin-induced pulmonary fibrosis in rats and the expression of metalloproteinase-9 and tissue inhibitors of metalloproteinase-1 in lung tissue. Chin Med J. 2014;127(7):1304-1309.

25. Xu T, Xie JY, Wang WM, Ren H, Chen N. Impact of rapamycin on peritoneal fibrosis and transport function. Blood Purif. 2012;34(1):48-57.

26. Yu SY, Liu L, Li P, Li J. Rapamycin inhibits the mTOR/p70S6K pathway and attenuates cardiac fibrosis in adriamycin-induced dilated cardiomyopathy. Thorac Cardiovasc Surg. 2013;61(3):223-228.

27. Chen $\mathrm{G}$, et al. Rapamycin ameliorates kidney fibrosis by inhibiting the activation of mTOR signaling in interstitial macrophages and myofibroblasts. PLoS One. 2012;7(3):e33626.

28. Zhu J, et al. Rapamycin inhibits hepatic stellate cell proliferation in vitro and limits fibrogenesis in an in vivo model of liver fibrosis. Gastroenterology. 1999;117(5):1198-1204.

29. Agarwal S, et al. Inhibition of Hifl $\alpha$ prevents both trauma-induced and genetic heterotopic ossification. Proc Natl Acad Sci U S A. 2016;113(3):E338-E347.

30. Ge Y, Chen J. Mammalian target of rapamycin (mTOR) signaling network in skeletal myogenesis. J Biol Chem. 2012;287(52):43928-43935.

31. Hagiwara H, Aida N, Machida J, Fujita K, Okuzumi S, Nishimura G. Contrast-enhanced MRI of an early preosseous lesion of fibrodysplasia ossificans progressiva in a 21-month-old boy. AJR Am J Roentgenol. 2003;181(4):1145-1147.

32. Kaplan FS, et al. Early diagnosis of fibrodysplasia ossificans progressiva. Pediatrics. 2008;121(5):e1295-e1300.

33. Joe AW, et al. Muscle injury activates resident fibro/adipogenic progenitors that facilitate myogenesis. Nat Cell Biol. 2010;12(2):153-163.

34. Uezumi A, et al. Identification and characterization of PDGFR $\alpha^{+}$mesenchymal progenitors in human skeletal muscle. Cell Death Dis. 2014;5:e1186. 
35. Uezumi A, et al. Fibrosis and adipogenesis originate from a common mesenchymal progenitor in skeletal muscle. J Cell Sci. 2011;124(Pt 21):3654-3664.

36. Rommel C, et al. Mediation of IGF-1-induced skeletal myotube hypertrophy by PI(3)K/Akt/mTOR and PI(3)K/Akt/GSK3 pathways. Nat Cell Biol. 2001;3(11):1009-1013.

37. Lee KW, et al. Rapamycin promotes the osteoblastic differentiation of human embryonic stem cells by blocking the mTOR pathway and stimulating the BMP/Smad pathway. Stem Cells Dev. 2010;19(4):557-568.

38. Ge Y, et al. mTOR regulates skeletal muscle regeneration in vivo through kinase-dependent and kinase-independent mechanisms. Am J Physiol, Cell Physiol. 2009;297(6):C1434-C1444.

39. Peterson JR, et al. Early detection of burn induced heterotopic ossification using transcutaneous Raman spectroscopy. Bone. 2013;54(1):28-34 